\title{
EMG-to-Force Modeling for Multiple Fingers
}

\author{
Pu Liu ${ }^{1}$, Donald R. Brown ${ }^{1}$, Francois Martel ${ }^{2}$, Denis Rancourt ${ }^{2}$ and Edward A. Clancy ${ }^{1}$ \\ ${ }^{1}$ Worcester Polytechnic Institute, 100 Institute Road, Worcester, MA 01609 U.S.A. \\ ${ }^{2}$ Université de Sherbrooke, 2500, boul. de l'Université Sherbrooke (Québec) CANADA J1K 2R1
}

\begin{abstract}
We provide a preliminary report on work to relate the EMG activity from forearm flexors and extensors to the flexionextension forces generated at the finger tips during constantposture, slowly force-varying contractions. EMG electrode arrays (up to 64 channels) were applied over the flexor and, separately, extensor musculature of the forearm. Spatial filters were used to create derived EMG channels that were then related to finger tip force (via least squares models). Preliminary results identify the "pinky" finger as having the most independent EMG-force control, with moderate control available from some combinations of the other fingers.
\end{abstract}

\section{INTRODUCTION}

Existing commercial EMG-controlled powered hand prostheses are limited to rudimentary control capabilities of either three discrete states (open, close, off) or one degree of freedom of proportional control [1]. Some studies of finger movement have considered multi-finger proportional control via EMG-based estimation of finger tip forces or finger joint angles [2], [3]. In this report, we describe preliminary findings of an exploratory study to relate forearm flexor and extensor EMG to flexion-extension force generated at the tips of the four fingers during constant-posture, slowly forcevarying contractions. A high resolution EMG array was utilized over the flexion and extension muscles of the forearm, and spatial filters were formed to enhance signal separation. The project goal was to assess the ability to determine two or more independent, continuous degrees of freedom of control from the antagonist muscles of the forearm.

\section{METHODS}

\section{A. Experimental Apparatus and Methods}

Experimental procedures were approved by the New England IRB. Subjects provided written informed consent. A custom-built restraint, shown in Fig. 1, was rigidly clamped to a table. The palm of the seated subject's hand was secured to the restraint with the thumb directed upwards, the four remaining digits were passively extended beyond the restraint and the elbow angle was $90^{\circ}$. The distal phalange of any one digit was secured to a load cell.

Two, 64-channel monopolar electrode arrays acquired the EMG (ELSCH064R3S Adhesive Electrode Arrays, EMGUSB Amplifier; OT Bioeletronica, Torino, Italy). Each array was a $13 \times 5$ matrix of electrodes (one corner electrode omitted), utilizing $2 \mathrm{~mm}$ diameter electrodes (gel-filled) separated by $8 \mathrm{~mm}$. The "flexion" array was oriented along

Supported by U.S. Army under USAMRAA Grant W81XWH-08-1-0422. the medial aspect of the forearm, the "extension" array along the lateral aspect. Eight extension electrodes were unused. Each electrode channel had a bandwidth from 10-750 Hz. EMG data were sampled at $2048 \mathrm{~Hz}$ (12-bits). A PC sampled the finger tip flexion-extension load cell data $(128 \mathrm{~Hz}$ sampling rate, 16 bits) and served as a subject display.

Four subjects completed one experiment. Each subject performed separate maximum voluntary contraction (MVC) flexion, then extension trials for each of the four digits. Thereafter, subjects performed a series of slowly forcevarying tracking trials, with their force ranging between $30 \%$ MVC extension and 30\% MVC flexion. Four tracking trials of $30 \mathrm{~s}$ duration were completed per digit.

\section{B. Methods of Analysis}

Data Preprocessing: Each monopolar EMG signal from the electrode arrays was band-pass filtered $(15-700 \mathrm{~Hz})$ and notch filtered at the power line frequency and all harmonics. Then, each trial was manually reviewed. EMG signals with anomalous data (e.g., obviously corrupted by excessive power line noise or motion artifact) were removed.

Formation of Classic Spatial Filters: Classic spatially filtered channels, using known (pre-selected) spatial filter weights [4], were formed. A spatial filter is a memory-less weighted sum of the monopolar signals. First, $L$ (preprocessed) monopolar signals were extracted for each of

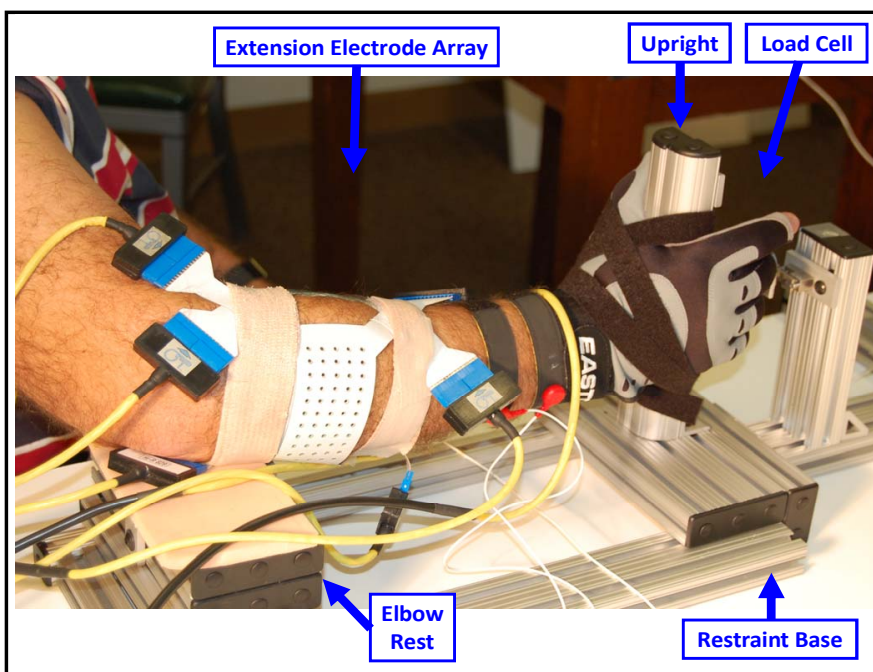

Fig. 1. Photograph of hand/arm secured into finger restraint. Velcro strap is wrapped around one finger (not visible) to secure it to the load cell. Gloved hand is held to the restraint upright using Velcro. Electrode arrays are mounted over the medial (flexion array-not visible) and lateral (extension array) aspects of forearm. 
the extensor and flexor muscle groups. These extracted channels served as a baseline set of spatially filtered channels. Separate such channel sets were formed for $L=13,7,5,4,3$ and 2 signals (spread transversely about the muscles). Second, bipolar channels and, third, linear double difference (LDD) channels were formed. Separate channel sets were again formed for $L=13,7,5,4,3$ and 2 signals. Lastly, normal double difference (NDD) filters were formed for $L=11,6,4,3$ and 2 . In total, 23 distinct spatially filtered channels were created for each of the flexor and extensor muscle groups.

EMG-Force Estimation: A separate EMG-force analysis was conducted for each of the 23 distinct spatially filtered channel sets. The EMG standard deviation (EMGб; a.k.a. EMG amplitude estimate) of each spatially filtered channel was computed and then decimated to $20.48 \mathrm{~Hz}$. The first and last five seconds of each $30 \mathrm{~s}$ tracking trial were discarded, to eliminate filter startup transients. Four trials, representing data from each of the four digits, were combined to form an analysis record. When one finger tip was active in the load cell, the finger tip force of the three remaining unmeasured finger tips was set to zero. Linear least squares was used to simultaneously relate the $L$ extension EMGo's and $L$ flexion EMGo's to the four finger tip forces. Separate training and testing records were used.

\section{PReliminary ReSUltS}

Only preliminary results are available at this time. Fig. 2 shows results using a bipolar montage of 13 derived electrode channels from each of the flexion and extension arrays. In these results, the "pinky" finger seems to exhibit excellent independent control and the "index" finger the least independent control. Some amount of EMG cross talk/muscle co-activation is visible in the EMG-force estimates for the index, middle and ring fingers.

Although statistical comparisons are not yet available, there did not seem to be an obvious advantage to use of the more complex spatial filter montages (LDD, NDD). One concern is that formation of these montages in software from monopolar electrodes is technically more challenging than doing so in hardware, and may lead to inferior comparisons.

\section{DISCUSSION}

In this study, we are concentrating on determining available degrees of freedom of independent, proportional control, expecting that future research would determine how those signals might be fully utilized to control a hand prosthesis. This study was intended as an initial assessment of EMGforce estimation in the finger tips. As such, several study limitations should be noted. First, data were only successfully collected from four subjects. Additional subjects would

improve generalizability of the results. Second, subjects only produced constant-posture, slowly force-varying contractions. Third, the performance of EMG-force models has seen little testing relative to the influences of localized muscle fatigue, electrode movement and day-to-day variations.

The electrode arrays used in this project are not appropriate for use in reusable systems (such as prosthetics) that are routinely donned and doffed by their user. The system was selected for its large number of active electrodes, with the understanding that knowledge learned in this study might direct research towards a more deployable electrode solution in the future. Future EMG-based prosthesis control systems might achieve high selectivity and better noise/interference performance via indwelling electrodes.

\section{REFERENCES}

[1] P. A. Parker, K. Englehart and B. Hudgins, "Myoelectric signal processing for control of powered limb prostheses," J. Electromyo. Kinesiol., vol. 16, pp.541-548, 2006.

[2] C. Castellini and P. van der Smagt, "Surface EMG in Advanced Hand Prosthetics," Bio. Cyber., vol. 100, pp. 35-47, 2009.

[3] R. J. Smith, D. Huberdeau, F. Tenore and N. V. Thakor, "Real-Time Myoelectric Decoding of Individual Finger Movements For a Virtual Target Task," Proc. 31st Ann. Int. Conf. IEE EMBS, 2009, pp. 23762379.

[4] C. Disselhorst-Klug, J. Bahm, V. Ramaekers, A. Trachterna and G. Rau, "Non-invasive approach of motor unit recording during muscle contractions in humans," Eur. J. Appl. Physiol., vol. 83, pp. 144-150, 2000. 